\title{
Exotic branes from del Pezzo surfaces
}

\author{
Justin Kaidi®* \\ Mani L. Bhaumik Institute for Theoretical Physics Department of Physics and Astronomy University of California, \\ Los Angeles, California 90095, USA
}

(Received 8 May 2019; published 23 September 2019)

\begin{abstract}
We revisit a correspondence between toroidal compactifications of M-theory and del Pezzo surfaces, in which rational curves on the del Pezzo are related to $\frac{1}{2}$-BPS branes of the corresponding compactification. We argue that curves of higher genus correspond to nongeometric backgrounds of the M-theory compactifications, which are related to exotic branes. In particular, the number of "special directions" of the exotic brane is equal to the genus of the corresponding curve. In addition to predicting three new nongeometric backgrounds, we discuss a relation between addition of curves in the del Pezzo and the brane polarization effect.
\end{abstract}

DOI: $10.1103 /$ PhysRevD.100.066025

\section{INTRODUCTION}

Despite their name, exotic branes are now understood to be ubiquitous and, due to the brane polarization effect, unavoidable even in traditional brane configurations [1,2]. A distinguishing feature of exotic branes is their unorthodox tension, which can scale as $g_{s}^{-\alpha}$ with $\alpha>2$ and which has distinguished behavior along some special directions. One denotes by $b_{\alpha}^{\left(c_{r}, \ldots, c_{2}\right)}$ the exotic brane with tension

$$
T_{p}\left(b_{\alpha}^{\left(c_{r}, \ldots, c_{2}\right)}\right)=\frac{R_{n_{1}} \ldots R_{n_{b-p}}}{g_{s}^{\alpha} \ell_{s}^{b+1}} \prod_{k=2}^{r}\left(\frac{R_{m_{1}^{(k)}} \ldots R_{m_{c_{k}}^{(k)}}}{\ell_{s}^{c_{k}}}\right)^{k}
$$

when the exotic brane world volume has $p+1$ noncompact directions (the maximum being $p+1=b+1$ ), $R_{i}$ are the radii of the internal directions, and $r \geq 2$.

One way to obtain exotic branes is to wrap traditional, higher-dimensional branes on compactification cycles and apply U-duality transformations to obtain objects which do not have any clear interpretation in terms of wrapped higher-dimensional branes [3,4]. An interesting question is to determine what exactly these exotic branes $d o$ correspond to in the uncompactified theory.

It has been argued $[1,2]$ that exotic branes have a higherdimensional origin as nongeometric backgrounds such as T-folds and U-folds, which extend the traditional notion of a manifold to allow for duality transformations in the

jkaidi@physics.ucla.edu

Published by the American Physical Society under the terms of the Creative Commons Attribution 4.0 International license. Further distribution of this work must maintain attribution to the author(s) and the published article's title, journal citation, and DOI. Funded by SCOAP ${ }^{3}$. transition functions between patches [5-9]. The fact that exotic branes involve nontrivial T- or U-duality monodromy means that they cannot be realized by any globally welldefined solutions of supergravity. Indeed, there may be configurations for which there is not even a local description in supergravity $[2,10]$. Instead, a complete description of these objects is expected to arise only after a manifestly T- or U-duality invariant formulation of the underlying theory has been obtained. One such formulation which has led to considerable progress is double field theory $[11,12]$ and its generalization to exceptional field theory [13-15]. Exotic branes have been successfully studied in these formalisms in e.g., $[10,16-20]$.

There is however another tentative U-duality invariant formulation of toroidal compactifications of M-theory, known as the "mysterious duality" [21-24]. In this "duality", M-theory on a rectangular torus $T^{k}$ with vanishing $C$-field vev is put into correspondence with the del Pezzo surface $\mathrm{dP}_{k}$. In addition to a map between moduli spaces, the correspondence suggests the identification of the spectrum of rational curves on $\mathrm{dP}_{k}$ (subject to some constraints to be reviewed below) with the spectrum of $\frac{1}{2}$-BPS branes in the $T^{k}$ compactification. One might naturally wonder whether the higher genus curves in $\mathrm{dP}_{k}$ also correspond to something in string theory. We will argue below that they correspond to nongeometric backgrounds, which can give rise to exotic branes upon further compactification.

\section{THE MYSTERIOUS DUALITY}

We begin by briefly reviewing the correspondence between $T^{k}$ compactifications of $\mathrm{M}$-theory and the $k$ th del Pezzo surface $\mathrm{dP}_{k}$. First, some basic facts about these surfaces will be needed. Recall that $\mathrm{dP}_{k}$ is $\mathbb{P}^{2}$ blown up at $k$-points, $k \leq 8$. For $\mathbb{P}^{2}$ there is a unique class of a curve 
$H \in H_{2}\left(\mathbb{P}^{2}, \mathbb{Z}\right)$. Since two lines generically intersect at a point, one has the intersection pairing $H \cdot H=1$. With each blow-up, one adds an exceptional curve $E_{i}$ such that $H_{2}\left(d P_{k}, \mathbb{Z}\right)=\operatorname{span}\left\{H, E_{1}, \ldots, E_{k}\right\}$, with the intersection pairings

$$
H \cdot H=1 \quad H \cdot E_{i}=0 \quad E_{i} \cdot E_{j}=-\delta_{i j}
$$

The canonical class for these surfaces is given by $K_{d P_{k}}=$ $-3 H+\sum_{i=1}^{k} E_{i}$.

On the other hand, Type IIB is put into correspondence with the Hirzebruch surface $\mathbb{F}^{0}=\mathbb{P}^{1} \times \mathbb{P}^{1}$, with compactification on $T^{k}$ again corresponding to the $k$ point blow-up, which we denote $\mathbb{F}^{k}$. The 2-cycles corresponding to the two $\mathbb{P}^{1}$ in $\mathbb{F}^{0}$ are denoted by $\ell_{1}, \ell_{2}$. We may think of one of the $\mathbb{P}^{1}$ as being trivially fibered over the other-since two fibers should not intersect one other, but each intersects the base once, we conclude that $\ell_{i} \cdot \ell_{j}=1-\delta_{i j}$. Each blowup adds an exceptional curve $e_{a}$, with the intersection pairings

$\ell_{i} \cdot \ell_{j}=1-\delta_{i j} \quad \ell_{i} \cdot e_{a}=0 \quad e_{a} \cdot e_{b}=-\delta_{\mathrm{ab}}$

The canonical class for these surfaces is $K_{\mathbb{F}^{k}}=-2 \ell_{1}-$ $2 \ell_{2}+\sum_{a=1}^{k} e_{a}$.

It is well known that $\mathbb{F}^{k} \cong \mathrm{dP}_{k+1}$; this is the del Pezzo analog of T-duality [21]. In particular, the bases of 2-cycles for the two surfaces are related by

$$
H \mapsto \ell_{1}+\ell_{2}-e_{1} \quad E_{1} \mapsto \ell_{2}-e_{1} \quad E_{2} \mapsto \ell_{1}-e_{1}
$$

as well as $E_{a+1} \mapsto e_{a}$ for the remaining exceptional curves. Furthermore, the invariance of $\mathbb{F}^{0}$ under exchange of $\ell_{1}$ and $\ell_{2}$ is interpreted as the invariance of Type IIB under S-duality.

In what sense do the del Pezzo surfaces "correspond" to the M-theory compactifications? First, there exists a map between the moduli spaces on the two sides. For rectangular M-theory compactifications with zero $C$-field vev, the moduli are the $k$ radii $R_{i}$ of the rectangular torus $T^{k}$ and the 11-dimensional Planck length $\ell_{p}$. These should be identified under those elements of the U-duality group which preserve the rectangular torus and vanishing $C$-field conditions, i.e., the Weyl group $\mathcal{W}\left(E_{k}\right) \subset E_{k(k)}(\mathbb{Z})$. The moduli space is then $\mathbb{R}_{+}^{k+1} / \mathcal{W}\left(E_{k}\right)$. For the del Pezzos, the moduli are the $k+1$ "volumes" $\left\{\omega(H), \omega\left(E_{i}\right)\right\}$ of the 2-cycles, ${ }^{1}$ subject to identification by those diffeomorphisms

\footnotetext{
${ }^{1}$ The generalized Kähler class $\omega \in H^{2}\left(d P_{k}, \mathbb{R}\right)$ is not unique and can in fact be shifted such that it is orthogonal to the canonical class. Indeed, we may decompose $\omega=\omega_{\perp}+\lambda K$ such that $\omega_{\perp} \cdot K=0$. The choice of parameter $\lambda$ corresponds to a choice of scale [21].
}

which leave the canonical class invariant. It is a remarkable fact that such diffeomorphisms are given by $\mathcal{W}\left(E_{k}\right)$ for $\mathrm{dP}_{k}$, thus reproducing the moduli space found before. The dictionary between the moduli proposed in [21] is ${ }^{2}$

$$
\omega(H) \leftrightarrow-3 \log \ell_{p} \quad \omega\left(E_{i}\right) \leftrightarrow-\log R_{i}
$$

Even more remarkable is the fact that the $\frac{1}{2}$-BPS branes in M-theory compactifications are in one-to-one correspondence with rational (i.e., genus zero) curves in the del Pezzo. Given such a rational curve $\mathcal{C} \in H_{2}\left(d P_{k}, \mathbb{Z}\right)$, we may calculate the tension $T_{p}$ and the worldvolume dimension $p+1$ of the corresponding brane via

$$
T_{p}=\exp \omega(\mathcal{C}) \quad p+1=d_{\mathcal{C}}
$$

In the above, $d_{\mathcal{C}}$ is the degree of the curve $\mathcal{C}$, defined as its intersection with the anticanonical class,

$$
d_{\mathcal{C}}=-K_{d P_{k}} \cdot \mathcal{C}
$$

For future reference, we note that the adjunction formula [25] allows us to relate $d_{\mathcal{C}}$ to the self-intersection and genus $g$ of $\mathcal{C}$ as follows,

$$
\mathcal{C} \cdot \mathcal{C}=2 g(\mathcal{C})-2+d_{\mathcal{C}}
$$

As an example of this "duality", consider uncompactified M-theory, which corresponds to $\mathbb{P}^{2}$. The latter has two genus zero curves $H$ and $2 H$, and a canonical class $K=-3 H$. Using the rules outlined in (5) and (6) we conclude that $H$ corresponds to a 2-brane of tension $\ell_{p}^{-3}$, while $2 H$ corresponds to a 5 -brane of tension $\ell_{p}^{-6}$. These correspond to the familiar M2 and M5 branes. We also see an example of a general phenomenon-branes which are electromagnetically dual correspond to rational curves $\mathcal{C}_{e}$ and $\mathcal{C}_{m}$ which are Serre dual, i.e.,

$$
\mathcal{C}_{e}+\mathcal{C}_{m}=-K
$$

This same procedure was repeated for Type IIA/B and compactifications thereof in [21], with agreement in all cases. For the uncompactified case, we list the set of rational curves and their corresponding tensions and string theory interpretations in Table I. Note importantly that we have required $0 \leq d_{\mathcal{C}} \leq 10$ to get a physical interpretation for the corresponding objects.

\footnotetext{
${ }^{2}$ For simplicity, we neglect factors of $2 \pi$ in formulas for volumes/tensions. Also, note that for $\mathbb{F}^{0} \leftrightarrow$ Type IIB the map is

$$
\omega\left(\ell_{1}\right) \leftrightarrow-2 \log \ell_{s} \quad \omega\left(\ell_{2}\right) \leftrightarrow-2 \log \ell_{s}-\log g_{s}
$$


TABLE I. Rational curves for $\mathrm{dP}_{1}$ and $\mathbb{F}^{0}$, together with their string theory interpretations.

\begin{tabular}{lcc}
\hline \hline Curve class & $T$ & Type IIA \\
\hline$E$ & $\ell_{s}^{-1} g_{s}^{-1}$ & D0 \\
$H-E$ & $\ell_{s}^{-2}$ & F1 \\
$H$ & $\ell_{s}^{-3} g_{s}^{-1}$ & D2 \\
$2 H-E$ & $\ell_{s}^{-5} g_{s}^{-1}$ & D4 \\
$2 H$ & $\ell_{s}^{-6} g_{s}^{-2}$ & NS5A \\
$3 H-2 E$ & $\ell_{s}^{-7} g_{s}^{-1}$ & D6 \\
$4 H-3 E$ & $\ell_{s}^{-9} g_{s}^{-1}$ & D8 \\
\hline \hline Curve class & $T$ & Type IIB \\
\hline$\ell_{1}$ & $\ell_{s}^{-2}$ & F1 \\
$\ell_{2}$ & $\ell_{s}^{-2} g_{s}^{-1}$ & D1 \\
$\ell_{1}+\ell_{2}$ & $\ell_{s}^{-4} g_{s}^{-1}$ & D3 \\
$2 \ell_{1}+\ell_{2}$ & $\ell_{s}^{-6} g_{s}^{-1}$ & D5 \\
$\ell_{1}+2 \ell_{2}$ & $\ell_{s}^{-6} g_{s}^{-2}$ & NS5B \\
$3 \ell_{1}+\ell_{2}$ & $\ell_{s}^{-8} g_{s}^{-1}$ & D7 \\
$\ell_{1}+3 \ell_{2}$ & $\ell_{s}^{-8} g_{s}^{-3}$ & 73 \\
$4 \ell_{1}+\ell_{2}$ & $\ell_{s}^{-10} g_{s}^{-1}$ & D9 \\
$\ell_{1}+4 \ell_{2}$ & $\ell_{s}^{-10} g_{s}^{-4}$ & 99 \\
\hline \hline & & \\
\hline
\end{tabular}

\section{EXOTIC BRANES}

In Table I we see already the appearance of exotic branes, albeit rather familiar ones - these are the $7_{3}^{0}$ and $9_{4}^{0} \mathrm{~B}$, which are the S-duals of D7 and D9 branes. Exotic branes will appear as rational curves whenever they are codimension $\leq 2$ in the compactification, as is the case for $7_{3}^{0}$ and $9_{4}^{0} \mathrm{~B}$ in uncompactified Type IIB. Instead of trying to catalogue all exotic branes obtained via compactification, our goal will be to obtain all 10- and 11-dimensional nongeometric backgrounds which reduce to the exotic branes upon appropriate compactification. We will see that these nongeometric backgrounds correspond to higher genus curves in the del Pezzos. Our strategy will be to compactify the theory, identify some codimension- 2 exotic branes, translate these branes to curves in the del Pezzo, and then take the blow-down (i.e., decompactification) limit.

Note that if $r=r^{\prime}$ is the largest number for which the index $c_{r^{\prime}}$ in $b_{\alpha}^{\left(c_{r}, \ldots, c_{2}\right)}$ is nonzero, we will refer to the exotic brane as an $\left(r^{\prime}-1\right)$-exotic brane. All traditional branes are 0 -exotic. Unless otherwise specified, we will be working with only 0 - and 1-exotic branes here. A more thorough analysis will be presented in upcoming work.

\section{A. M-theory and Type IIA}

Let us begin by compactifying M-theory or Type IIA on $T^{4}$ or $T^{3}$ to $d=7$. From the string theory point of view, it is known that there are 20 codimension- $2 \frac{1}{2}$-BPS objects in $d=7$, including thirteen 1-exotic branes and seven wrapped D-branes [2]. In the del Pezzo picture, these must correspond to rational curves $\mathcal{C} \in H_{2}\left(d P_{4}, \mathbb{Z}\right)$ such that $d_{\mathcal{C}}=5$. A generic element of $H_{2}\left(d P_{4}, \mathbb{Z}\right)$ may be written as $\mathcal{C}=m H-n E-\sum_{i=1}^{3} n_{i} E_{i}$. Note that we have singled out one of the exceptional curves $E$-from the perspective of M-theory $E$ is on equal footing with the other $E_{i}$, but from the Type IIA perspective $E$ will be taken to be the M-theory circle, i.e., the second element of $H_{2}\left(d P_{1}, \mathbb{Z}\right)$. Making use of (8), we have the following constraints on $\left\{m, n, n_{i}\right\}$,

$$
3 m-n-\sum_{i=1}^{3} n_{i}=5 \quad m^{2}-n^{2}-\sum_{i=1}^{3} n_{i}^{2}=3
$$

One finds 20 integers solutions to these constraints. The resulting curves and the corresponding codimension-2 objects in $d=7$ M-theory and Type IIA are shown in Table II. We see that we have obtained a number of exotic objects, including e.g., the Kaluza-Klein monopole KK5A, which in standard notation is $52 \mathrm{~A}$

Having identified these exotic branes with particular curves in the del Pezzo, we would now like to understand where they originate from in the uncompactified theory. From the del Pezzo point of view, the decompactification limit simply corresponds to blowing down the exceptional curves. Beginning with the case of Type IIA, we blow down the $E_{i}$ and see that $5_{2}^{2} \mathrm{~A}$ descends from the curve $4 H-2 E \in H_{2}\left(d P_{1}, \mathbb{Z}\right)$, the $4_{3}^{3}$ descends from $4 H-E$, the KK5A descends from the anticanonical class $-K_{d P_{1}}=$ $3 H-E$, and the $6_{3}^{1}$ descends from $3 H$. These curves were not included in Table I since they are not rational-rather they have genus 2, 3, 1, and 1 respectively. It is these higher genus curves that we claim correspond to the nongeometric backgrounds underlying exotic branes.

Because we began by considering only rational curves in $d=7$, we have obtained only a subset of the possible nongeometric backgrounds. We may continue this analysis by compactifying to lower dimensions $d \geq 3$, leading to the following general conclusion. For a curve $\mathcal{C}_{m n}=m H-n E$ on $\mathrm{dP}_{1}$, we first define the exotic brane noncompact world volume dimension as

$$
b_{\mathcal{C}}+1=d_{\mathcal{C}}-2 g(\mathcal{C})
$$

which is the generalization of (6) to higher genus. Then for every curve $\mathcal{C}_{m n}$ (subject to restrictions given momentarily) we obtain a nongeometric background,

$$
\mathcal{C}_{m n} \in H_{2}\left(d P_{1}, \mathbb{Z}\right) \leftrightarrow\left[\left(b_{\mathcal{C}_{m n}}\right)_{m-n}^{g\left(\mathcal{C}_{m n}\right)}\right] .
$$

The brackets on the right-hand side indicate that we are really referring to equivalence classes of higher-dimensional nongeometric backgrounds, labeled by their lowerdimensional exotic branes. The elements in each equivalence 
TABLE II. Rational curves for $\mathrm{dP}_{4}$ and their $d=7$ M-theory and Type IIA interpretations. The multiplicities of configurations are shown in bold. Note that all of the branes above are codimension-2, e.g., D6 represents a twice-wrapped D6.

\begin{tabular}{lccc}
\hline \hline Curve class & $T_{4}$ & M-theory & Type IIA \\
\hline $2 H-E_{i}$ & $R_{i} \ell_{p}^{-6} \sim R_{i} \ell_{s}^{-6} g_{s}^{-2}$ & M5 (3) & NS5 (3) \\
$2 H-E$ & $R_{M} \ell_{p}^{-6} \sim \ell_{s}^{-5} g_{s}^{-1}$ & M5(1) & D4(1) \\
$3 H-E_{i}-E_{j}-2 E_{k}$ & $R_{i} R_{j} R_{k}^{2} \ell_{p}^{-9} \sim R_{i} R_{j} R_{k}^{2} \ell_{s}^{-9} g_{s}^{-3}$ & KK6 (3) & $6_{3}^{1}(\mathbf{3})$ \\
$3 H-2 E-E_{i}-E_{j}$ & $R_{i} R_{j} R_{M}^{2} \ell_{p}^{-9} \sim R_{i} R_{j} \ell_{s}^{-7} g_{s}^{-1}$ & KK6 (3) & D6 (3) \\
$3 H-E-E_{i}-2 E_{j}$ & $R_{M} R_{i} R_{j}^{2} \ell_{p}^{-9} \sim R_{i} R_{j}^{2} \ell_{s}^{-8} g_{s}^{-2}$ & KK6 (6) & KK5A (6) \\
$4 H-E-2 E_{1}-2 E_{2}-2 E_{3}$ & $R_{M}\left(R_{1} R_{2} R_{3}\right)^{2} \ell_{p}^{-12} \sim\left(R_{1} R_{2} R_{3}\right)^{2} \ell_{s}^{-11} g_{s}^{-3}$ & $5^{3}(\mathbf{1})$ & $4_{3}^{3}(\mathbf{1})$ \\
$4 H-2 E-E_{i}-2 E_{j}-2 E_{k}$ & $R_{i}\left(R_{M} R_{j} R_{k}\right)^{2} \ell_{p}^{-12} \sim R_{i}\left(R_{j} R_{k}\right)^{2} \ell_{s}^{-10} g_{s}^{-2}$ & $5^{3}(\mathbf{3})$ & $5_{2}^{2} \mathrm{~A}(\mathbf{3})$ \\
\hline \hline
\end{tabular}

class have the same del Pezzo origin-for example, one has $\left[7_{3}^{(1,0)}\right] \sim\left[4_{3}^{3}\right]$ in Type IIA and $\left[7_{3}^{(2,0)}\right] \sim\left[4_{3}^{(1,3)}\right] \sim\left[1_{3}^{6}\right]$, $\left[6_{3}^{(1,1)}\right] \sim\left[3_{3}^{4}\right]$ in Type IIB.

With the map (12), we may now classify all backgroundsgeometric and nongeometric - which descend to $\frac{1}{2}$-BPS traditional and 1-exotic branes in Type IIA. These are labeled by $\mathcal{C}_{m n}$ with $m, n \in \mathbb{Z}$ such that

$$
\begin{aligned}
& b_{\mathcal{C}_{m n}}+1 \geq 0 \quad g\left(\mathcal{C}_{m n}\right) \geq 0 \\
& b_{\mathcal{C}_{m n}}+g\left(\mathcal{C}_{m n}\right)+1 \leq 10 .
\end{aligned}
$$

We list all possibilities in Table III, indexed by the power $\alpha$ of $g_{s}$ in the tension formula, $T \sim g_{s}^{-\alpha}$. In the current case, we have $\alpha=m-n$.

Before moving on to Type IIB, we first describe the situation for M-theory. Decompactification now involves blow-down of $E$ as well. The exotic brane $5^{3}$ is then seen to descend from the curve $4 H \in H_{2}\left(\mathbb{P}^{2}, \mathbb{Z}\right)$, while the KK6 descends from the anticanonical class $-K_{\mathbb{P}^{2}}=3 H$. These curves are again not rational-they are of genus 3 and 1 , respectively. More generally, given a curve $\mathcal{C}_{n}=n H$, we claim that there is a corresponding class of nongeometric backgrounds in M-theory,

$$
\mathcal{C}_{n} \in H_{2}\left(\mathbb{P}^{2}, \mathbb{Z}\right) \leftrightarrow\left[\left(b_{\mathcal{C}_{n}}\right)^{g\left(\mathcal{C}_{n}\right)}\right]
$$

TABLE III. (Non)geometric backgrounds descending to (exotic) branes in Type IIA/B with tension $T \sim g_{s}^{-\alpha}$.

\begin{tabular}{ccc}
\hline \hline$\alpha$ & Type IIA & Type IIB \\
\hline 0 & {$\left[1_{0}^{0}\right]$} & {$\left[1_{0}^{0}\right]$} \\
1 & {$\left[0_{1}^{0}\right],\left[2_{1}^{0}\right],\left[4_{1}^{0}\right],\left[6_{1}^{0}\right],\left[8_{1}^{0}\right]$} & {$\left[(-1)_{1}^{0}\right],\left[1_{1}^{0}\right],\left[3_{1}^{0}\right],\left[5_{1}^{0}\right],\left[7_{1}^{0}\right],\left[9_{1}^{0}\right]$} \\
2 & {$\left[5_{2}^{\ell} \mathrm{A}\right] \ell \in\{0, \ldots, 4\}$} & {$\left[5_{2}^{\ell} \mathrm{B}\right] \ell \in\{0, \ldots, 4\}$} \\
3 & {$\left[6_{3}^{1}\right],\left[4_{3}^{3}\right],\left[2_{3}^{5}\right],\left[0_{3}^{7}\right]$} & {$\left[7_{3}^{0}\right],\left[5_{3}^{2}\right],\left[3_{3}^{4}\right],\left[1_{3}^{6}\right],\left[(-1)_{3}^{8}\right]$} \\
4 & {$\left[9_{4}^{0} \mathrm{~A}\right],\left[5_{4}^{3} \mathrm{~A}\right],\left[1_{4}^{6} \mathrm{~A}\right]$} & {$\left[9_{4}^{0} \mathrm{~B}\right],\left[5_{4}^{3} \mathrm{~B}\right],\left[1_{4}^{6} \mathrm{~B}\right]$} \\
5 & {$\left[2_{5}^{6}\right]$} & {$\left[5_{5}^{4}\right],\left[(-1)_{5}^{8}\right]$} \\
6 & $\cdots$ & $\cdots$ \\
7 & {$\left[0_{7}^{9}\right]$} & $\cdots$ \\
\hline \hline
\end{tabular}

labeled by 1 -exotic branes so long as (13) is satisfied. ${ }^{3}$ In fact, these conditions restrict us to $n \in\{1, \ldots, 5\}$, which give five corresponding (non)geometric backgrounds $\left[2^{0}\right]$, $\left[5^{0}\right],\left[6^{1}\right],\left[5^{3}\right]$, and $\left[2^{6}\right]$. This reproduces the standard M2 and M5 branes, the KK6, and the two known 1-exotic brane backgrounds $[17,19]$.

\section{B. Type IIB}

We may repeat the above exercise for Type IIB. We begin by considering the theory compactified on $T^{3}$. The spectrum of $\frac{1}{2}$-BPS codimension- 2 objects may be obtained by T-dualizing the Type IIA spectrum presented above. Recall that at the level of the del Pezzo, T-duality just amounts to a change of basis 2-cycles, as shown in (3). Implementing this change of basis and using (4), we obtain the spectrum of curves and corresponding branes shown in Table IV.

One may again obtain the (non)geometric backgrounds in higher dimensions corresponding to each of these by tuning the exceptional curves to zero. Thus for example we conclude that the exotic brane $5_{2}^{2} \mathrm{~B}$ descends from a tendimensional nongeometric background corresponding to the cycle $3 \ell_{1}+2 \ell_{2}$, while the uplift of the exotic brane $5_{3}^{2}$ corresponds to the cycle $2 \ell_{1}+3 \ell_{2}$. Recalling that S-duality is implemented by exchange of $\ell_{1}$ and $\ell_{2}$, we also conclude that $\left[5_{2}^{2} \mathrm{~B}\right] \stackrel{S}{\leftrightarrow}\left[5_{3}^{2}\right]$, as is known $[17,19]$. As before, these examples may be generalized by considering further compactifications, leading to the proposal that any curve $\mathcal{C}_{m n}=m \ell_{1}+n \ell_{2}$ corresponds to a class of nongeometric backgrounds,

$$
\mathcal{C}_{m n} \in H_{2}\left(\mathbb{F}^{0}, \mathbb{Z}\right) \leftrightarrow\left[\left(b_{\mathcal{C}_{m n}}\right)_{n}^{g\left(\mathcal{C}_{m n}\right)}\right]
$$

labeled by 1-exotic branes as long as it satisfies (13). We list all possibilities in Table III, indexed by the power $\alpha$ of $g_{s}$ in the tension formula, $T \sim g_{s}^{-\alpha}$. In the current case, we see that $\alpha=n$.

\footnotetext{
${ }^{3}$ The right-hand side of the final line of (13) is now 11.
} 
TABLE IV. Rational curves for $\mathbb{F}^{3} \cong \mathrm{dP}_{4}$ and their $d=7$ Type IIB interpretations. Multiplicities are shown in bold. All of the branes above are codimension- 2 .

\begin{tabular}{lcc}
\hline \hline Curve class & $T_{4}$ & Type IIB \\
\hline$\ell_{1}+2 \ell_{2}-e_{i}$ & $R_{i} \ell_{s}^{-6} g_{s}^{-2}$ & NS5 (3) \\
$2 \ell_{1}+\ell_{2}-e_{i}$ & $R_{i} \ell_{s}^{-6} g_{s}^{-1}$ & D5 (3) \\
$2 \ell_{1}+2 \ell_{2}-2 e_{i}-e_{j}$ & $R_{i}^{2} R_{j} \ell_{s}^{-8} g_{s}^{-2}$ & KK5B (6) \\
$3 \ell_{1}+\ell_{2}-e_{1}-e_{2}-e_{3}$ & $R_{1} R_{2} R_{3} \ell_{s}^{-8} g_{s}^{-1}$ & D7 (1) \\
$\ell_{1}+3 \ell_{2}-e_{1}-e_{2}-e_{3}$ & $R_{1} R_{2} R_{3} \ell_{s}^{-8} g_{s}^{-3}$ & $7_{3}^{0}(\mathbf{1})$ \\
$3 \ell_{1}+2 \ell_{2}-e_{i}-2 e_{j}-2 e_{k}$ & $R_{i}\left(R_{j} R_{k}\right)^{2} \ell_{s}^{-10} g_{s}^{-2}$ & $5_{2}^{2} \mathrm{~B}(\mathbf{3})$ \\
$2 \ell_{1}+3 \ell_{2}-e_{i}-2 e_{j}-2 e_{k}$ & $R_{i}\left(R_{j} R_{k}\right)^{2} \ell_{s}^{-10} g_{s}^{-3}$ & $5_{3}^{2}(\mathbf{3})$ \\
\hline \hline
\end{tabular}

\section{Remarks}

We now remark on the results in Table III. At orders $\alpha=0,1$ we obviously reproduce the familiar geometric backgrounds corresponding to the fundamental string and $\mathrm{D} p$-branes. At order $\alpha=2$, we obtain the NS5 backgrounds $\left[5_{2}^{0} \mathrm{~A} / \mathrm{B}\right]$, the KK-monopoles $\left[5_{2}^{1} \mathrm{~A} / \mathrm{B}\right]$, as well as three other nongeometric backgrounds. Their existence has already been argued for-they correspond to the 1-exotic branes completing the T-duality orbit $5_{2}^{0} \mathrm{~A} / \mathrm{B} \stackrel{T}{\leftrightarrow} 5_{2}^{1} \mathrm{~B} /$ $\mathrm{A} \stackrel{T}{\leftrightarrow} 5_{2}^{2} \mathrm{~A} / \mathrm{B} \stackrel{T}{\leftrightarrow} 5_{2}^{3} \mathrm{~B} / \mathrm{A} \stackrel{T}{\leftrightarrow} 5_{2}^{4} \mathrm{~A} / \mathrm{B}[10,20]$.

The nongeometric backgrounds found at order $\alpha=3,4$, 5 capture all 1-exotic branes known in the literature [17,19]. The 0 -exotic codimension- 0 object $\left[9_{4}^{0} \mathrm{~B}\right]$ has the correct tension to be the S-dual of the D9 brane [26]. In addition, the del Pezzo curve classification predicts a pair of nongeometric backgrounds $\left[(-1)_{3}^{8}\right] \stackrel{S}{\leftrightarrow}\left[(-1)_{5}^{8}\right]$ of Type IIB. The $\left[(-1)_{3}^{8}\right]$ fits together with the known results via T-duality $(-1)_{3}^{8} \stackrel{T}{\leftrightarrow} 00_{3}^{7}$, though this is not true for $\left[(-1)_{5}^{8}\right]$ at the level of 1 -exotic branes. The representative 1-exotic branes of these two nongeometric backgrounds have not appeared in previous catalogues $[17,19,27]$ since those works studied only exotic branes appearing in dimensions $d \geq 3$, whereas these new 1-exotic branes only appear upon compactification to $d=2$. We are further led to propose that these are the only additional 1-exotic branes appearing at $d=2$.

Likewise, at $\alpha=7$ the del Pezzo analysis predicts a nongeometric background $\left[0_{7}^{9}\right]$ in Type IIA, corresponding to the cycle $6 H+E \in H_{2}\left(d P_{1}, \mathbb{Z}\right)$. This does not correspond to any known exotic brane, nor is it connected via T-duality to any Type IIB nongeometric background which may be labeled by a 1-exotic brane. It appears as a 1-exotic brane only at $d=1$.

We close this section by mentioning that, as in the original work on the M-theory/del Pezzo correspondence, we have imposed by hand a restriction (13) on the curves of the del Pezzo in order to get a sensible physical interpretation. It would be interesting to see whether the curves violating these constraints could also be given some interpretation in string and M-theory.

\section{BRANE POLARIZATION}

In this final section we ask what addition of curves corresponds to in string theory. Given classes $\mathcal{C}_{m_{1} n_{1}}$ and $\mathcal{C}_{m_{2} n_{2}}$, we may use (12) or (15) to obtain the Type IIA/B backgrounds corresponding to the $\operatorname{sum} \mathcal{C}_{m_{1}+m_{2}, n_{1}+n_{2}}$. These formulas imply that objects of tension $T_{i} \sim g_{s}^{-\alpha_{i}}$ add to an object of tension $T \sim g_{s}^{-} \sum_{i} \alpha_{i}$. This is the behavior expected of branes undergoing brane polarization [2].

To have brane polarization, one often requires a specific relative arrangement and angular momentum for the branes participating in the process - this data is not yet known to be captured by the del Pezzo. However, the del Pezzo correspondence suggests that if a certain brane polarization is possible, it must be between a set of branes whose corresponding curves are related by addition. We will require that (13) be satisfied by the $\operatorname{sum} \mathcal{C}_{m_{1}+m_{2}, n_{1}+n_{2}}$.

We now give some simple examples involving two branes polarizing to a third. We begin with Type IIA, where the curves have the form $\mathcal{C}_{m n}=m H-n E$. By Table I, one finds

$$
\mathcal{C}_{0,-1}+\mathcal{C}_{1,1} \sim \mathcal{C}_{1,0} \Rightarrow[\mathrm{D} 0]+[\mathrm{F} 1] \sim[\mathrm{D} 2] .
$$

This is the usual supertube effect [28]. A more exotic example is

$$
\mathcal{C}_{2,1}+\mathcal{C}_{2,1} \sim \mathcal{C}_{4,2} \Rightarrow[\mathrm{D} 4]+[\mathrm{D} 4] \sim\left[5_{2}^{2} \mathrm{~A}\right] .
$$

Of course we could also have a polarization involving more than one brane on the right-hand side, e.g.,

$\mathcal{C}_{2,1}+\mathcal{C}_{2,1} \sim \mathcal{C}_{3,1}+\mathcal{C}_{1,1} \Rightarrow[\mathrm{D} 4]+[\mathrm{D} 4] \sim[\mathrm{KK} 5 \mathrm{~A}]+[\mathrm{F} 1]$.

These two spontaneous polarizations can be obtained by dualizing the supertube effect, and were discussed e.g., in [2]. On the other hand, note that $\mathcal{C}_{0,-1}$ and $\mathcal{C}_{1,0}$ only add to give $\mathcal{C}_{1,-1}$, which has negative virtual genus. Thus we expect no spontaneous polarization of an isolated D0 and D2 to a third brane.

In Type IIB with curves $\mathcal{C}_{m n}=m \ell_{1}+n \ell_{2}$, we give the following two examples,

$$
\begin{aligned}
& \mathcal{C}_{0,1}+\mathcal{C}_{2,1} \sim \mathcal{C}_{2,2} \Rightarrow[\mathrm{D} 1]+[\mathrm{D} 5] \sim[\mathrm{KK} 5 \mathrm{~B}] \\
& \mathcal{C}_{1,1}+\mathcal{C}_{1,2} \sim \mathcal{C}_{2,3} \Rightarrow[\mathrm{D} 3]+[\mathrm{NS} 5] \sim\left[5_{3}^{2}\right] .
\end{aligned}
$$

The first of these gives the Lunin-Mathur geometries $[29,30]$. More generally, since we have found that the KK-monopole always corresponds to the anticanonical class, by (9) we conclude that electromagnetically dual branes can always polarize to a KK-monopole. The second process above is discussed in [2].

\section{ACKNOWLEDGMENTS}

We thank Eric D'Hoker and Ben Michel for discussions. 
[1] J. de Boer and M. Shigemori, Phys. Rev. Lett. 104, 251603 (2010).

[2] J. de Boer and M. Shigemori, Phys. Rep. 532, 65 (2013).

[3] N. A. Obers and B. Pioline, Phys. Rep. 318, 113 (1999).

[4] E. A. Bergshoeff and F. Riccioni, J. High Energy Phys. 01 (2018) 046.

[5] C. M. Hull, J. High Energy Phys. 10 (2005) 065.

[6] C. M. Hull, J. High Energy Phys. 07 (2007) 080.

[7] A. Kumar and C. Vafa, Phys. Lett. B 396, 85 (1997).

[8] J. T. Liu and R. Minasian, Nucl. Phys. B510, 538 (1998).

[9] J. X. Lu and S. Roy, Nucl. Phys. B538, 149 (1999).

[10] R. Otsuki, D. S. Berman, and E. T. Musaev, arXiv:1903 .10247.

[11] C. Hull and B. Zwiebach, J. High Energy Phys. 09 (2009) 099.

[12] G. Aldazabal, D. Marques, and C. Nunez, Classical Quantum Gravity 30, 163001 (2013).

[13] O. Hohm and H. Samtleben, Phys. Rev. Lett. 111, 231601 (2013).

[14] O. Hohm and H. Samtleben, Phys. Rev. D 89, 066016 (2014).

[15] O. Hohm and H. Samtleben, Phys. Rev. D 89, 066017 (2014).

[16] C. D. A. Blair and E. Malek, J. High Energy Phys. 03 (2015) 144.
[17] I. Bakhmatov, D. Berman, A. Kleinschmidt, E. Musaev, and R. Otsuki, J. High Energy Phys. 08 (2018) 021.

[18] D. S. Berman, E. T. Musaev, and R. Otsuki, J. High Energy Phys. 12 (2018) 053.

[19] J. J. Fernández-Melgarejo, T. Kimura, and Y. Sakatani, J. High Energy Phys. 09 (2018) 072.

[20] T. Kimura, S. Sasaki, and K. Shiozawa, J. High Energy Phys. 07 (2018) 001.

[21] A. Iqbal, A. Neitzke, and C. Vafa, Adv. Theor. Math. Phys. 5, 769 (2001).

[22] P. Henry-Labordere, B. Julia, and L. Paulot, J. High Energy Phys. 04 (2002) 049.

[23] P. Henry-Labordere, B. Julia, and L. Paulot, arXiv:hep-th/ 0303178.

[24] P. Henry-Labordere, B. Julia, and L. Paulot, J. High Energy Phys. 04 (2003) 060.

[25] P. Griffiths and J. Harris, Principles of Algebraic Geometry (John Wiley \& Sons Inc., New York, 1994).

[26] C. M. Hull, Nucl. Phys. B509, 216 (1998).

[27] A. Kleinschmidt, J. High Energy Phys. 10 (2011) 144.

[28] D. Mateos and P. K. Townsend, Phys. Rev. Lett. 87, 011602 (2001).

[29] O. Lunin and S. D. Mathur, Nucl. Phys. B623, 342 (2002).

[30] O. Lunin, J. M. Maldacena, and L. Maoz, arXiv:hep-th/ 0212210. 